\title{
Frontières
}

\section{Le motif du Christ mort dans la photographie contemporaine}

\section{Cadavres et figures endormies}

\section{Nathalie Dietschy}

Volume 23, numéro 2, printemps 2011

Enquêtes sur le cadavre : 2. Fantastique

URI : https://id.erudit.org/iderudit/1007586ar

DOI : https://doi.org/10.7202/1007586ar

Aller au sommaire du numéro

\section{Éditeur(s)}

Université du Québec à Montréal

ISSN

1916-0976 (numérique)

Découvrir la revue

Citer cet article

Dietschy, N. (2011). Le motif du Christ mort dans la photographie contemporaine : cadavres et figures endormies. Frontières, 23(2), 26-32. https://doi.org/10.7202/1007586ar
Résumé de l'article

Le cadavre le plus représenté dans l'art occidental est celui de Jésus. Le Christ mort est en effet un thème iconographique qui a été maintes fois interprété en peinture, en sculpture et plus récemment en photographie. Les artistes contemporains s'emparent de ce thème pour en donner une vision personnelle et profane. Deux tendances sont à distinguer. La première relève de postures christomorphes de modèles ou d'artistes qui identifient leurs propres souffrances à celle du crucifié. La seconde démarche est héritière de l'art du passé, mais s'en éloigne pour destituer la figure christique en banalisant sa mort à travers des photographies de modèles endormis qui imitent des tableaux célèbres. Le présent article se propose d'analyser ces deux points de vue actuels portés sur ce qui constitue un sujet majeur de l'histoire de l'art. 


\section{Résumé}

Le cadavre le plus représenté dans l'art occidental est celui de Jésus. Le Christ mort est en effet un thème iconographique qui a été maintes fois interprété en peinture, en sculpture et plus récemment en photographie. Les artistes contemporains s'emparent de ce thème pour en donner une vision personnelle et profane. Deux tendances sont à distinguer. La première relève de postures christomorphes de modèles ou d'artistes qui identifient leurs propres souffrances à celle du crucifié. La seconde démarche est héritière de l'art du passé, mais s'en éloigne pour destituer la figure christique en banalisant sa mort à travers des photographies de modèles endormis qui imitent des tableaux célèbres. Le présent article se propose d'analyser ces deux points de vue actuels portés sur ce qui constitue un sujet majeur de l'histoire de l'art.

Mots-clés: Jésus-cadavre-Christ mortphotographie contemporaine-sommeil.

\section{Abstract}

The corpse the most represented in Western art is Christ's dead body. Indeed, the Dead Christ is an iconographic theme that has been interpreted many times in painting as much as in sculpture and most recently in photography. Contemporary artists encounter this iconography to give a personal and secular point of view. Two tendencies must be pointed out. The first one are Christ-like poses that can be understood as identifications with Christ's suffering. The second tendency overtly reveals its legacy to old art, but subverts it to degrade the Christ figure, making Jesus' death ordinary in pictures that show sleeping models in attitudes that are codified and related to traditional representations of the Dead Christ. This article intends to analyze these two contemporary points of view on a major subject of art history.

Keywords: Jesus-corpse-Dead Christcontemporary photography-sleep.

\section{LE MOTIF \\ DU CHRIST MORT DANS \\ LA PHOTOGRAPHIE CONTEMPORAINE Cadavres et figures endormies}

\footnotetext{
Nathalie Dietschy, cand. Ph.D., assistante diplômée,

Centre des sciences historiques de la culture, Département d'Histoire de l'art, Université de Lausanne (Suisse).
}

Dans l'histoire de l'art occidental, le cadavre le plus représenté est sans nul doute celui de Jésus. L'épisode de sa mort marque pour le croyant une étape importante, puisqu'elle signifie que le Christ a accepté son destin tragique en vue du salut de l'humanité. Une fois décédé et descendu de croix, le Christ, dont la double origine, divine et humaine, est toute particulière, est ramené à sa condition terrestre. Son cadavre est ainsi corruptible comme celui de tout être humain. Depuis des siècles, si la crucifixion demeure le thème privilégié des artistes qui ont à dessein de représenter la souffrance endurée par le crucifié, de nombreuses œuvres présentent l'épisode après la sentence, celle qui exhibe le cadavre de Jésus. Quatre épisodes principaux suivent celui de la crucifixion: la descente de croix, la déposition dans laquelle Jésus décloué est ramené au sol et enveloppé d'un linceul blanc, la lamentation où le défunt est pleuré par les proches, et la mise au tombeau de la dépouille, déposée dans un sépulcre neuf ${ }^{1}$. D'autres scènes en sont dérivées: la pietà constitue une image intimiste se concentrant sur la tristesse souvent contenue de la Vierge tenant son fils mort sur ses genoux, et le Christ mort exhibe le cadavre, quelques instants avant l'embaumement.

Depuis la fin des années 1980 et le début des années 1990, les thèmes chrétiens, ceux de la Passion et notamment du Christ mort $^{2}$, se multiplient dans l'art contemporain $^{3}$. Deux tendances peuvent être dégagées quant à l'usage du motif du Christ mort. La première consiste à associer des photographies de malades, de mourants, de postures de souffrance, prises dans un cadre séculier, à l'iconographie du thème du Christ mort. Les artistes mettent en scène leur sujet à la manière des peintures traditionnelles de ce topos, identifiant les douleurs du siècle à celles du crucifié. La seconde tendance s'appuie également sur l'héritage iconographique religieux, mais 
le détourne pour opérer une banalisation, une «profanisation ${ }^{4}$. Ces deux points de vue contemporains qui se basent sur ce que l'on peut nommer une démarche d'imitatio Christi, divergent dans leur interprétation et révèlent le regard porté sur la représentation du cadavre de Jésus.

\section{CADAVRES CHRISTOMORPHES}

La photographie de Thérèse Frare, David Kirby's Final Moments (1990), montre un homme à l'agonie. David Kirby, trente-deux ans, malade du sida, vit ses derniers instants accompagné de sa famille. Sa maigreur cadavérique témoigne des ravages du virus. La mise en scène du sidéen sur son lit de mort assisté des siens renvoie spontanément à la représentation traditionnelle de la lamentation, image du Christ défunt pleuré par la Vierge, MarieMadeleine et l'apôtre Jean. L'agencement de la photographie réactive dans l'inconscient collectif le souvenir des multiples tableaux de la dépouille du Christ entouré de ses proches. L'œuvre de Frare-qui n'a pas intitulé son cliché pietà, celui-ci ayant été désigné ainsi a posteriori-fait ouvertement référence au thème chrétien.

Dans les années 1980, le sida se propage et bouleverse la conception de l'amour et de la sexualité qui devient potentiellement meurtrière. Plusieurs artistes sont touchés par le virus ou leur entourage et évoquent cette pandémie dans leurs travaux, à l'instar de Ron Athey (1961), artiste américain qui apprend sa séropositivité en 1986 et qui, depuis lors, assimile son calvaire à celui du crucifié ou au supplice de saint Sébastien criblé de flèches (voir Dietschy, 2011). "J'ai été touché par le complexe du martyr bien avant le sida, avoue Athey, mais le sida a été si dramatique dans ma vie, à la fin des années 1980, que j'ai été forcé d'incarner le martyr d'un saint dans mon travail artistique» (Liotard, 2003, p. 117). La photographie prise par Catherine Opie (1961), Ron Athey/The Sick Man (de la performance Deliverance, 2000), témoigne de l'identification opérée par Ron Athey. Ce dernier imite la posture du Christ à l'agonie, soutenu par un homme nu tatoué, dans une version personnelle de la pietà. Athey se projette dans la figure de l'Homme de Douleur, dont il assimile la souffrance à son état de contaminé et interprète sa séropositivité comme un véritable chemin de croix.

L'autofiction de Ron Athey qui incarne un martyr contemporain est aussi à l'œuvre dans la mise en scène de l'agonie de David Kirby qui, même si elle atteste d'un événement authentique - un témoignage de la mort de cet activiste de la cause homosexuelle-, n'en demeure pas moins une image dont la composition ajoute une

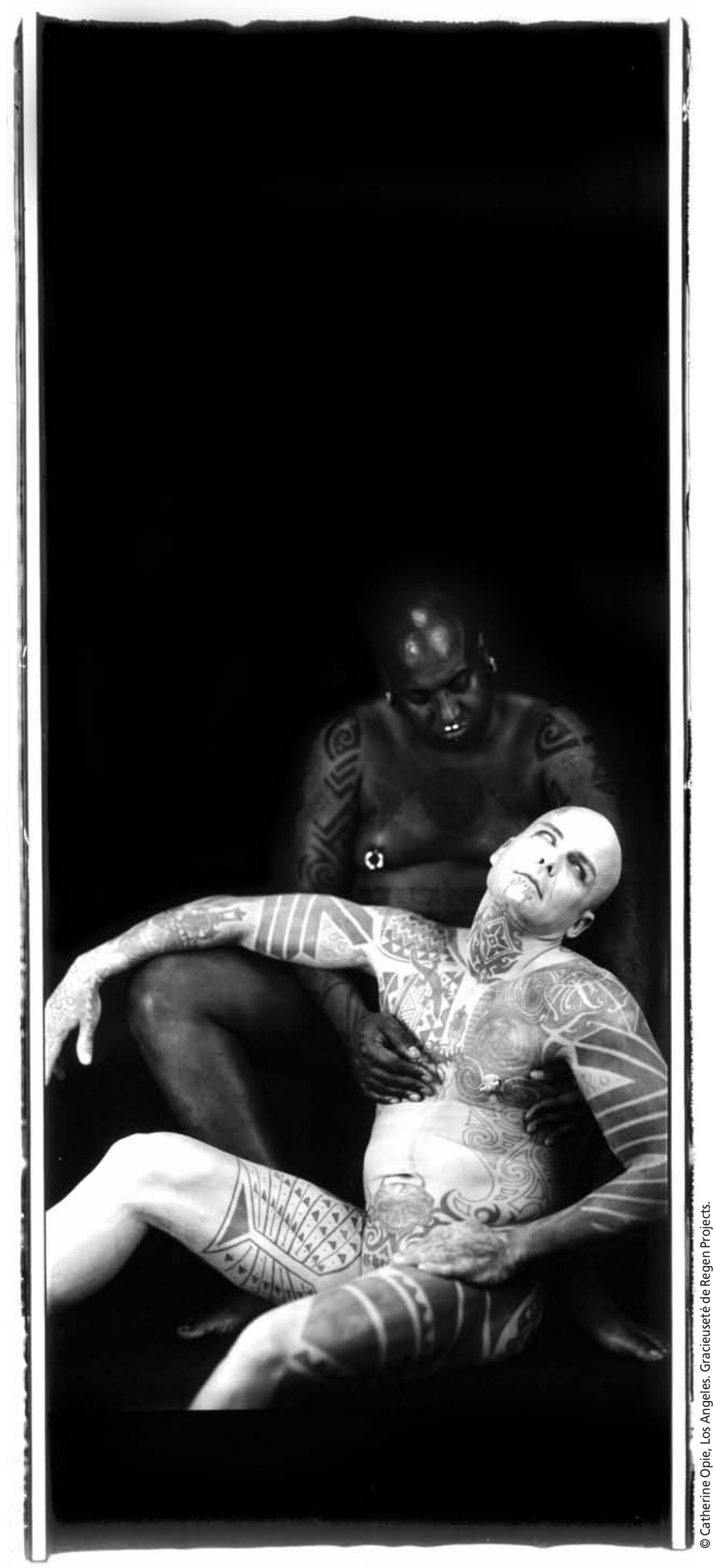

Catherine Opie, Ron Athey/The Sick Man (de la performance Deliverance), 2000, polaroïd, édition unique, $279,4 \times 104,1 \mathrm{~cm}$. 


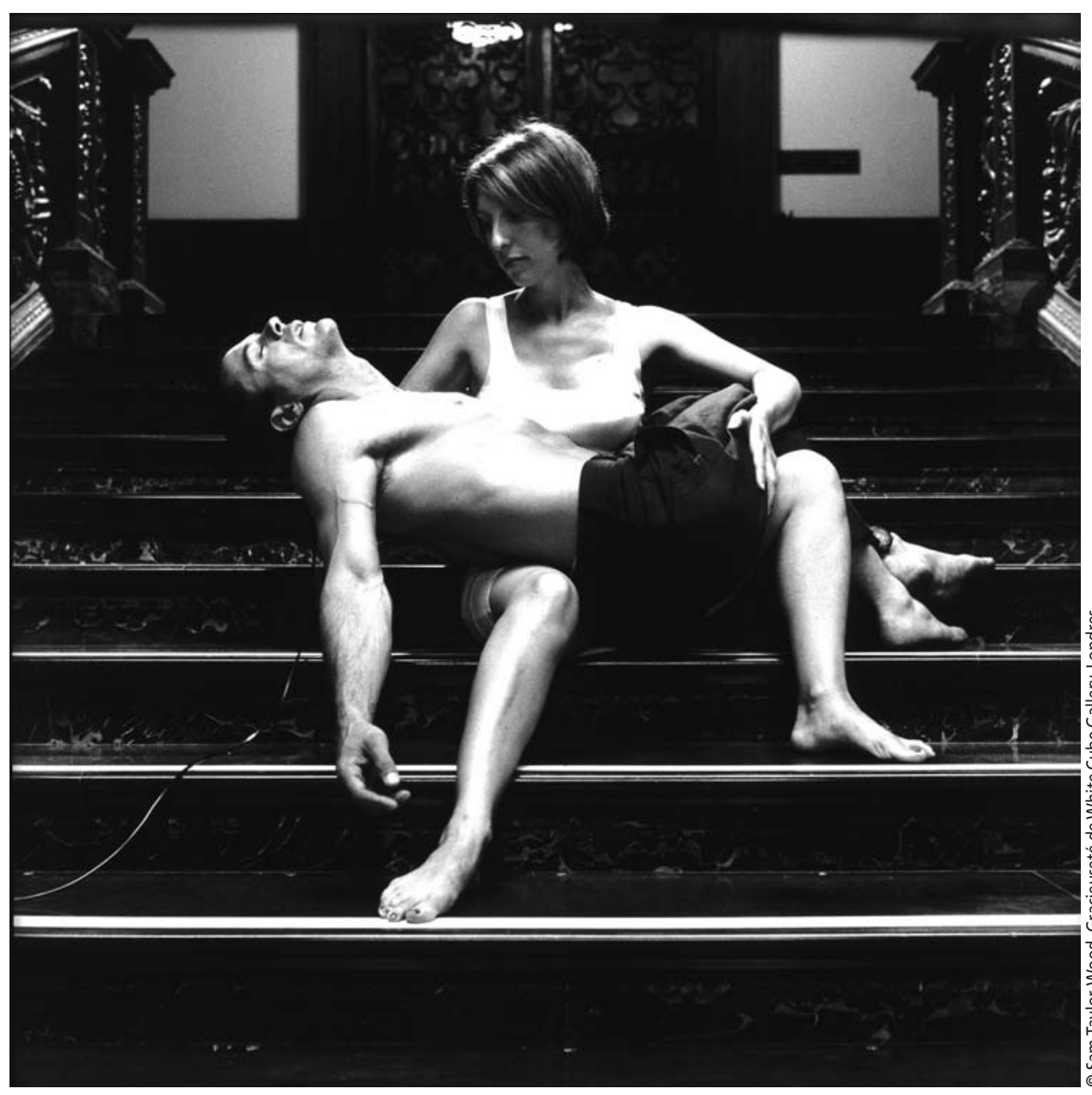

Sam Taylor-Wood, Self Pietà, 2001, C-print, 134,5 x 134,5 x 4,5 cm (encadré).

dimension symbolique qui dépasse le seul aspect documentaire. David Kirby mourant est présenté à la manière du Christ mort dépeint dans les tableaux de la lamentation hérités de la Renaissance, moyen pour la photographe d'élever ce décès particulier au rang d'icône. Le cliché de Thérèse Frare n'est pas seulement celui d'un homme qui va rendre son dernier souffle, il est avant tout celui de la mort dans ce qu'elle a d'universel, d'implacable. L'œuvre de Frare, par l'association formelle à la lamentation, hisse la mort individuelle de Kirby en emblème universel des conséquences tragiques de la maladie.

En 1992, la marque de vêtements Benetton utilise le tirage de Frare pour ses affiches publicitaires ${ }^{5}$. L'usage commercial de l'image d'un sidéen crée la polémique dans le monde entier. Des associations venant en aide aux malades considèrent que l'œuvre donne une vision effrayante de l'épidémie, alors que d'autres s'offusquent de l'association entre l'agonie de Kirby et la mort du Christ. Oliviero Toscani, publicitaire sulfureux de la marque de vêtements, confie qu'il a choisi l'œuvre de Frare en raison de sa connotation chrétienne. Il insiste sur le fait que, contrairement aux représentations traditionnelles du Christ mort, ladite photographie montre une «vraie pietà» (Rutherford, 2000, p. 161). David Kirby incarne ainsi une nouvelle «image» du Christ mort, sacrifié sur l'autel de l'épidémie de la fin du $\mathrm{XX}^{\mathrm{e}}$ siècle. Ron Athey se projette, de son côté, dans la figure christique pour traduire la souffrance de celui qui se sait condamné, véritable Homme de Douleur actuel. Ces identifications révèlent la profonde influence exercée par l'iconographie chrétienne que les photographes s'approprient afin de traduire des drames personnels.

En 2001, l'artiste britannique Sam Taylor-Wood (1967) se photographie assise sur les marches d'un escalier, tenant sur ses genoux son ami, l'acteur américain Robert Downey Junior. Self Pietà, comme son titre l'indique, constitue une version subjective du thème et s'inspire librement de la célèbre Pietà (1498-99) ${ }^{6}$ de Michel-Ange. Le tirage est dérivé d'une vidéo qu'elle a réalisée sur le sujet. On y voit le couple sur le même escalier, l'artiste s'efforçant de retenir son compagnon pour l'empêcher de tomber (Pietà, 2001) ${ }^{7}$. Les légers mouvements de l'acteur américain-qui aurait pu feindre sans difficulté l'immobilité parfaite d'un cadavre-dévoilent la fiction de la mise en scène tout comme son corps musclé et les veines gonflées de son bras tombant à terre témoignent que l'acteur n'a rien du physique rachitique du crucifié. Ces infidélités au thème traduisent la symbolique de l'œuvre: l'artiste tente de soutenir son ami qui venait de suivre une cure de désintoxication. Le cliché de Taylor-Wood s'inscrit dans le courant actuel de la staged photography ${ }^{8}$ dont les procédés relèvent des tableaux vivants de l'époque victorienne qui reproduisaient sur une scène les attitudes et les poses de peintures traditionnelles, parfois immortalisées par l'appareil photographique. Taylor-Wood et son modèle rejouent la pietà devant l'objectif, mais le mimétisme est approximatif. Ils n'imitent pas exactement les postures canoniques du thème, mais le citent par le biais de références ancrées dans le savoir collectif. L'artiste qui a été guérie d'un cancer, utilise le motif de la pietà comme une pathos formulae (Warburg, 1990), une formule visuelle à la forte charge pathétique qui permet de toucher le spectateur qui reconnaît immédiatement le sujet représenté. À travers le motif de la pietà qu'elle familiarise, elle évoque les épreuves que l'acteur et elle ont dû surmonter.

Sam Taylor-Wood opère le mouvement inverse de Thérèse Frare qui avait rendu la mort de David Kirby universelle et atemporelle, en la hissant au rang d'«icône profane». Taylor-Wood s'empare, elle, du motif religieux de la pietà pour le singulariser, l'insérer dans son quotidien et celui de ses proches. D'ailleurs, l'artiste n'adopte pas une expression de chagrin et l'acteur ne joue pas véritablement au mort. La pose des deux modèles les promeut, il est vrai, au rang de martyrs, mais cette identification a pour dessein d'exhiber la vulnérabilité de l'artiste et de son modèle qui ont tous deux combattu la maladie. La pietà demeure, selon Taylor-Wood, «un modèle toujours opérationnel parce que correspondant à des sentiments et à des événements restés intangibles depuis deux millénaires, soit à une nature essentielle de l'humain qui réside au-delà des aléas de l'histoire»(Grenier, 2003, p. 17). Taylor-Wood et Downey Jr. dévoilent leurs souffrances intimes; l'artiste et l'acteur remplacent le culte des figures bibliques par celui de l'individualité.

En 2000, l'artiste né à Londres, Lee Wagstaff (1969) se photographie dans une œuvre dont le titre renvoie à la symbolique christique. Dans un décor neutre sombre, Lamb présente l'artiste couché, portant un tissu blanc autour des hanches qui rappelle 
le perizonium du crucifié. La composition est héritière du célèbre tableau du Christ mort (c. 1480) ${ }^{9}$ d'Andrea Mantegna. Le cadavre de Jésus y est peint allongé sur une table de marbre. La perspective raccourcie met l'accent sur ses pieds aux stigmates. Le vase utilisé pour l'embaumement, ainsi que la plaque brute sur laquelle le corps a été déposé installent la scène dans une morgue, quelques instants avant la mise au tombeau. Mantegna a pris soin de décrire l'épisode comme une mort clinique, réaliste, détaillant les traces des meurtrissures du crucifié, dessinant méticuleusement les orifices créés par les clous lors de la crucifixion. Les proches, relégués sur le côté, à peine visibles, viennent se recueillir sur le corps sans vie de Jésus dont les plaies ont été nettoyées avant l'inhumation. La perspective choisie par Mantegna souligne non pas le chagrin que provoque le deuil, mais les traces physiques de la torture, puisque les pieds, placés au premier plan, sont littéralement mis en avant. Wagstaff a surélevé le point de fuite du chef-d'œuvre de la Renaissance, installant ses deux pieds au centre de son cliché qui ne permet pas de distinguer son visage. Les deux stigmates du Britannique ne sont pas des blessures, mais deux tatouages. L'artiste nu s'exhibe en cadavre, dans une autofiction qui le présente à la manière du Christ mort et dévoile deux motifs de svastika inscrits à l'encre bleue dans sa peau. La croix à branches, qui a été détournée par Hitler sous le Troisième Reich (la croix gammée est dextrogyre, contrairement au motif originel qui est lévogyre), constitue un symbole cyclique bouddhiste et hindouiste

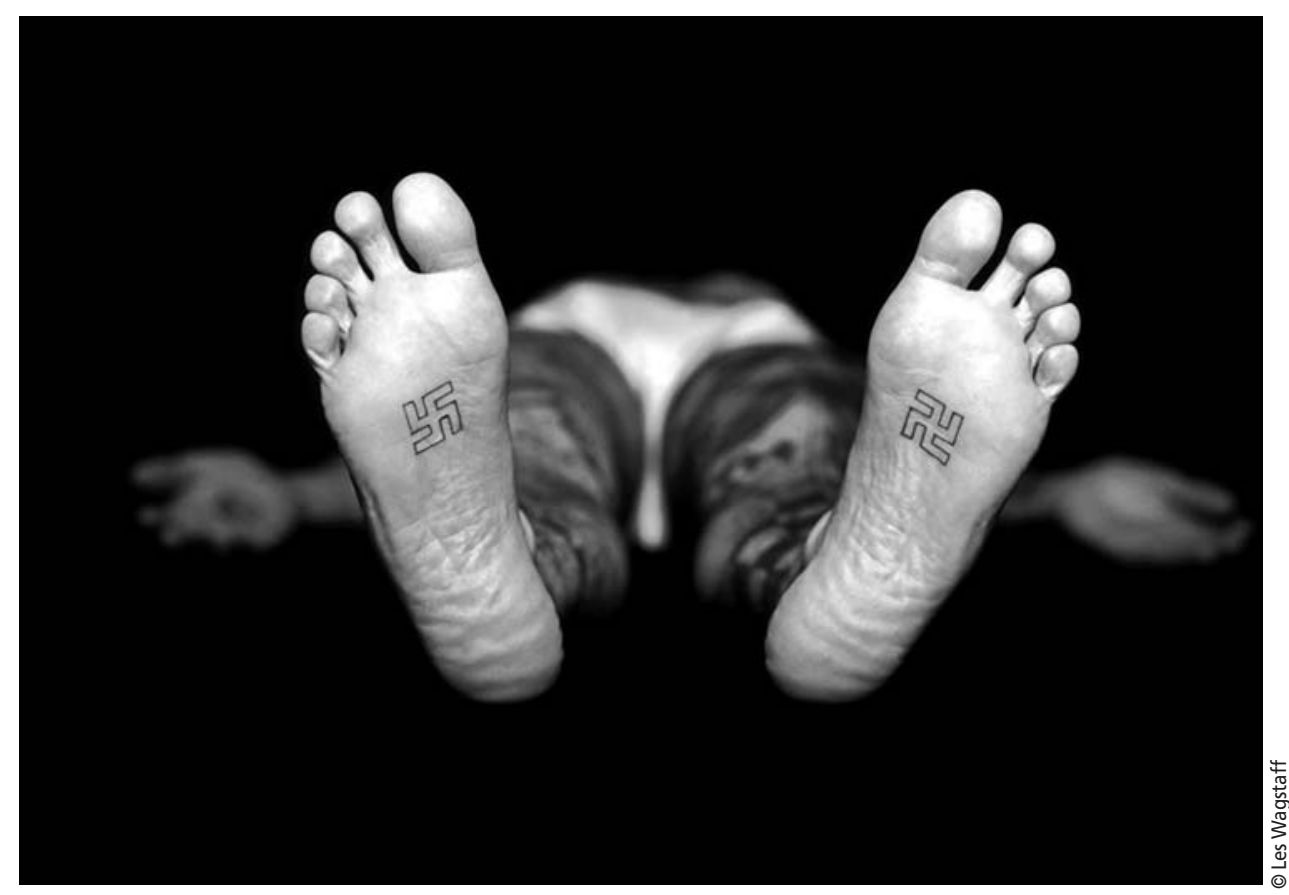

Lee Wagstaff, Lamb, 2000, C-print, 153 x $188 \mathrm{~cm}$.
INSÉRÉ DANS LE MONDE PROFANE, LE THÈME CHRÉTIEN,

QU'IL S'AGISSE DU CHRIST MORT, DE LA PIETÀ OU DE LA CRUCIFIXION,

EST DÉSACRALISÉ ET ÉVOQUE DES AFFECTS PUREMENT HUMAINS,

CONFINANT PARFOIS À L'ORDINAIRE.

et il n'est pas rare de le voir sur la poitrine de Bouddha ${ }^{10}$. Wagstaff mêle un symbole venu d'Asie, marquant ainsi l'influence de sa famille paternelle indienne, à une représentation chrétienne, provenant de son héritage catholique maternel. L'artiste tente de dépasser les clivages culturels et force le spectateur à adopter un regard inédit sur les diverses traditions. À travers cet autoportrait christique, l'artiste interroge le rapport de chacun aux motifs et aux symboles et questionne l'influence des différentes cultures et de l'Histoire. Il mime le Christ mort de Mantegna et s'expose dans le rôle d'un cadavre, reflet de soi peu banal. La référence au code iconographique chrétien relève aussi de sa conception du tatouage, qu'il associe à une pratique religieuse. Dans une série de photographies intitulée Apostles (2005), Wagstaff réalise des portraits d'hommes tatoués sur l'entier de leur torse, identifiant chacun d'eux à un apôtre et incarnant lui-même le Christ. «Je me suis intéressé à l'idée que le tatouage est comme une religion », explique-t-il. «Elle exige beaucoup d'engagement, de détermination et a un effet durable sur les personnes qui en portent. Beaucoup de modèles de la série sont des tatoueurs au style très différent, qu'ils transmettent

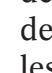
les yeux fermés. Complètement nu, il est couché sur un lit aux draps blancs, dans une pièce vide dont on perçoit une porte à l'arrière-plan. La composition à l'horizontalité accentuée cite explicitement le célèbre Christ mort (1521) ${ }^{14}$ peint par Hans Holbein et conservé à Bâle. Le chefd'œuvre de la Renaissance souligne le réalisme de la mort de Jésus dont la maigreur et les blessures sont effrayantes. La main crispée, l'œil livide, la peau gris-bleu aux extrémités du corps attestent de la progression du processus de décomposition du cadavre. Holbein soumet au spectateur une image terrible, anatomique et brutale de la mort. L'oppressante horizontalité de la composition rappelle les tombeaux placés dans les églises, où une vitre latérale permet aux fidèles de se recueillir devant le corps sculpté du Christ défunt.

La photographe et vidéaste britannique recycle le thème chrétien pour l'installer dans un cadre profane: le modèle ne porte 


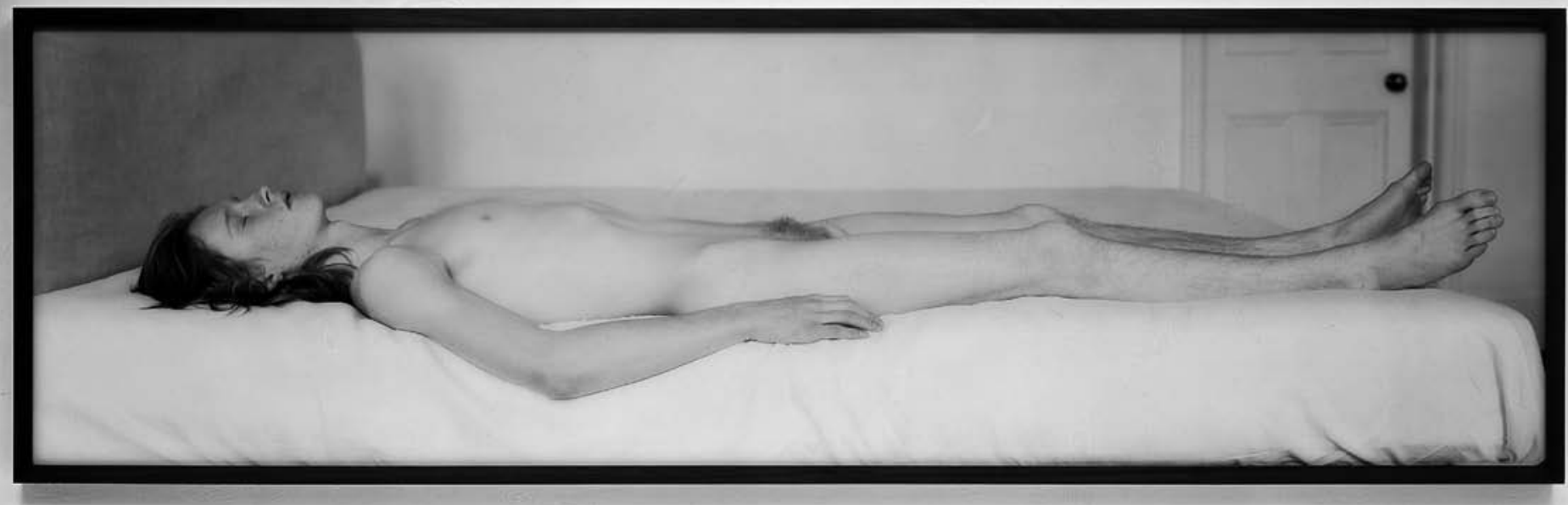

Sam Taylor-Wood, Sleep, 2002, C-print, 75 × 246 cm (encadré).

aucun stigmate, il n'est pas un cadavre, mais il fait simplement une sieste. Cependant, la force du modèle holbeinien s'impose et conditionne la lecture de l'œuvre. Sam Taylor-Wood met en scène ce «sommeil de la mort » shakespearien que décrit Hamlet dans son célèbre soliloque:

For in that sleep of death

what dreams may come,

When we have shuffled off

this mortal coil,

Must give us pause $[\ldots]^{15}$.

Le monologue de Hamlet se retrouve également dans une série de neuf clichés intitulée Soliloquy. Le soliloque, monologue intérieur du discours de soi à soi, est mis en image à travers la décomposition de chaque œuvre en deux parties distinctes, à la manière des tableaux d'autel. Dans le panneau principal est présenté le sujet pensant et dans la partie inférieure, sa pensée, ses rêves ou fantasmes. Agencée comme un retable, Soliloquy VII (1999), qui cite le célèbre Christ mort de Mantegna, montre un parc arborisé dans la prédelle et un homme couché sur un lit dans la partie principale, grandeur nature. Le modèle de Taylor-Wood, s'il imite fidèlement sa référence iconographique, en diffère par l'absence de stigmates. L'homme n'est pas mort, mais simplement endormi. Allongé et complètement nu, il rêve au paysage affiché sous ses pieds, dans la prédelle.

Inséré dans le monde profane, le thème chrétien, qu'il s'agisse du Christ mort, de la pietà ou de la crucifixion, est désacralisé et évoque des affects purement humains, confinant parfois à l'ordinaire. Cette reprise formelle de tableaux célèbres paraît trop manifeste selon Dominique Baqué. L'auteure déplore chez l'artiste anglaise un «appropriationnisme ostentatoire», «une excessive monstration des références culturelles» (Baqué, 2001, p. 79; Baqué, 2004, p. 108). Sam Taylor-Wood ne s'approprie cependant pas le code iconographique du Christ mort par pur principe de recyclage postmoderne; elle détourne la mort en sommeil et exprime, à travers les références qu'elle assume, la difficulté d'être, d'«être ou ne pas être ${ }^{16}$. L'artiste explore les mécanismes de la pensée, les

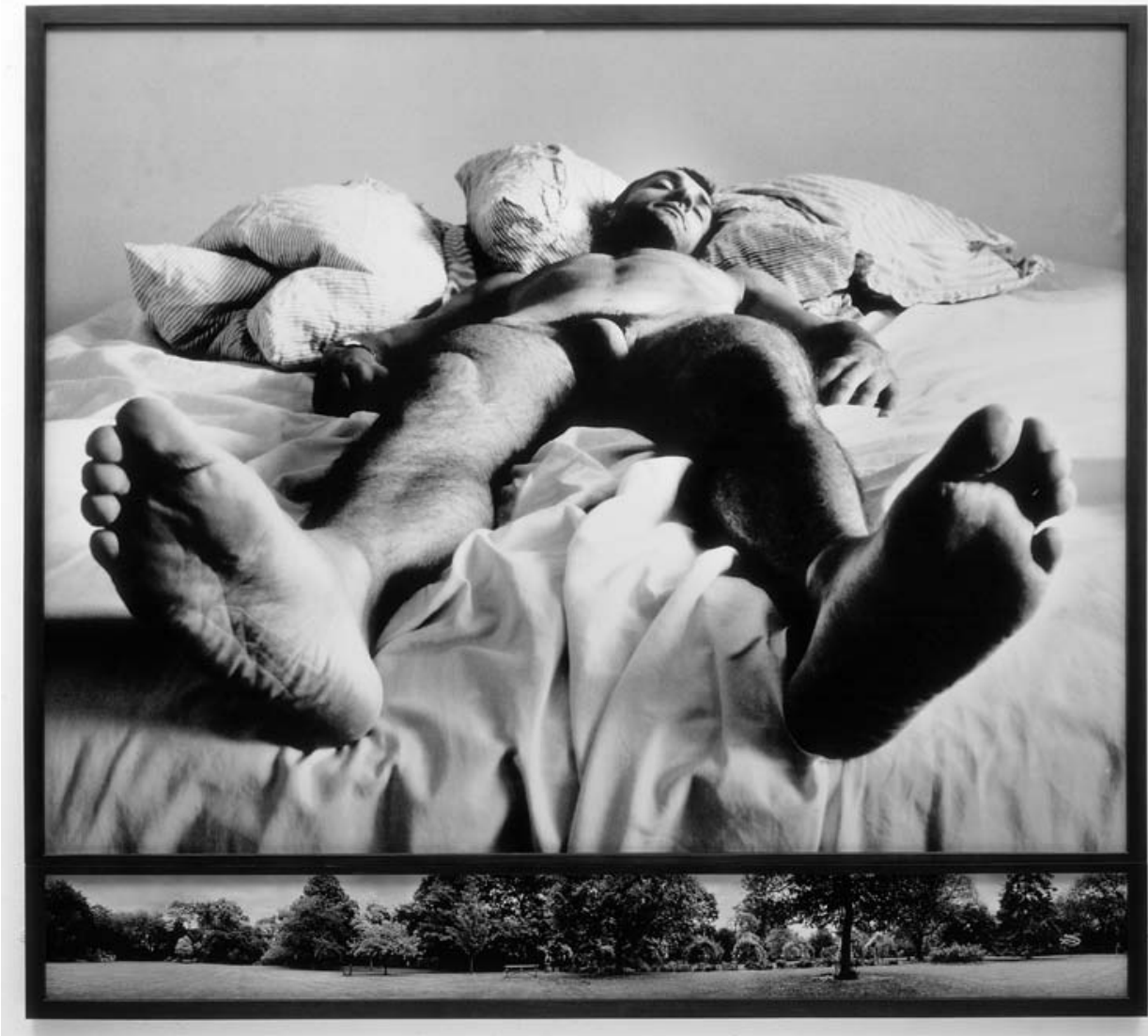

Sam Taylor-Wood, Soliloquy VII, 1999, C-print, 222 × 242 (encadré en deux parties). rêves et désirs refoulés qui se dévoilent dans les "prédelles» de chacun des soliloques, des monologues qui dialoguent avec la tradition picturale et littéraire (Dondero, 2009).

Sam Taylor-Wood s'inscrit dans un courant actuel qui multiplie les reprises de chefs-d'œuvre et notamment des tableaux du Christ mort de Mantegna et de Holbein, à l'instar de Bettina Rheims 
(1952) qui s'inspire également de l'œuvre de Mantegna. Sa version, qui fait partie de sa série photographique de portée confessionnelle ${ }^{17}$ I.N.R.I. $(1998)^{18}$, conserve le même agencement, mais son modèle couché a été maquillé en mort, teint pâle et profondes plaies sur la plante des pieds. Le spectateur n'est pourtant pas dupe: le mannequin engagé par la photographe française pour la prise de vue n'a pas le corps émacié de Jésus. Si le projet de Bettina Rheims a pour dessein de souligner que le Christ est mort pour l'humanité, celui de Sam Taylor-Wood dévalorise la portée symbolique de l'épisode en transformant la mort en banal assoupissement. Cette tendance à la «profanisation » de la figure christique se retrouve dans une œuvre du photographe britannique Paul Hodgson (1972), qui cite également le thème peint par Mantegna. Dans Rehearsal (2001) ${ }^{19}$, une femme est assise à côté d'un homme couché sur un lit, photographié selon la même perspective que le tableau dont il s'inspire. La femme lui tourne le dos, le regard triste. Le quotidien morose se montre comme un épisode biblique dramatique, par le biais des références iconographiques. Sans stigmate, le modèle allongé n'a rien d'un cadavre : il dort. Le titre indiquant qu'il s'agit d'une "répétition », d'un jeu mimétique comme au théâtre, assume l'héritage pictural de la photographie, la mise en scène « répétée» devant l'objectif.

\section{HYPNOS ET THANATOS}

Les références à l'iconographie traditionnelle chrétienne dans un cadre profane sont aujourd'hui courantes et manifestent non seulement l'influence de ces canons, mais aussi leur capacité à être transposés dans le quotidien contemporain. La forte charge pathétique de la pietà sert à exprimer les tourments de Sam Taylor-Wood, tout comme la composition de la prise de vue de Thérèse Frare emploie l'agencement archétypal de la lamentation pour hisser le drame individuel d'un sidéen au rang d'icône tragique de l'épidémie. La projection de ces situations particulières dans une iconographie religieuse a pour effet d'émouvoir le spectateur qui reconnaît aisément l'épisode chrétien auquel l'œuvre fait allusion. Le parallèle entre les stigmates christiques et les tatouages de svastikas réalisé par Lee Wagstaff, qui porte sur son torse un tatouage de Sacré-Cœur enveloppant une croix à branches, exprime les diverses influences de l'artiste qui tente de s'émanciper des clivages culturels et religieux. L'identification de Ron Athey à saint Sébastien ou au Christ mort participe de cette même tendance à la familiarisation du récit biblique. L'artiste malade qui se perçoit comme un martyr, s'approprie la figure de l'Homme de Douleur, comparant son calvaire de séropositif au chemin de croix. Le deuil, le chagrin, la douleur et la maladie que ces photographies expriment, sont traduits à travers la mise en scène de codes iconographiques associant ces tragédies à la mort du Christ.

D'autres œuvres, en revanche, semblent destituer le décès de Jésus en le transformant en simple sommeil. L'homme endormi de Paul Hodgson pose bien à la manière du Christ mort, mais la citation n'est que formelle, le modèle n'a plus rien de christique. Quant aux versions de Sam Taylor-Wood montrant des figures en pleine sieste dans des mises en scène reprises de l'iconographie traditionnelle religieuse, elles interprètent le motif chrétien de manière profane, laissant la possibilité de rêver à diverses transpositions subjectives, car comme le dit Hamlet, "mourir, dormir, / Dormir, rêver peutêtre $^{20} » \ldots$

\section{Bibliographie}

ASHLEY (2005). "An interview with Lee Wagstaff », Skin Deep, n 128, décembre.

BAL, M. (1999). Quoting Caravaggio: Contemporary Art, Preposterous History, Chicago, University of Chicago Press.

BAQUÉ, D. (2001). "Sam Taylor-Wood», Art Press, $\mathrm{n}^{\circ} 273$, novembre, p. 79.

BAQUÉ, D. (2004). Photographie plasticienne: L'extrême contemporain, Paris, éd. du Regard.

BARROERO, S. (2001), « Rencontre autour du thème de la Chair et Dieu. Interview de Lee Wagstaff », entretien réalisé le 200105-23, en ligne, <http://www.artistes-endialogue.org/wags03.htm>, consulté le 2011-09-01.

BELTING, H. (2007). La Vraie image, Paris, Gallimard.

DIETSCHY, N. (2008). "L'Autoportrait en Christ de Renee Cox: étude d'un scandale», dans J. KAEMPFER, Ph. KAENEL, A. BOILLAT et P. GISEL (dir.), Études de Lettres, "Points de vue sur Jésus au $\mathrm{XX}^{\mathrm{e}}$ siècle », $\mathrm{n}^{\circ}$ 2, p. 145-167.

DIETSCHY, N. (2011). "Ecce homo: Jésus, l'artiste et l'homosexualité dans la photographie contemporaine », dans A. BOILLAT, Ph. KAENEL et J. KAEMPFER (dir.), Jésus en représentations, Gollion, Infolio.

DONDERO, M.G. (2009). Le Sacré dans l'image photographique: études sémiotiques, Paris, Hermès Science, Lavoisier.

FOLIE, S. et M. GLASMEIER (2002). Tableaux vivants: Lebende Bilder und Attitüden in Fotografie, Film und Video, cat. d'expo., Vienne, Kunsthalle, 24 mai au 25 août 2002, Wien, Kunsthalle Wien.

GRENIER, C. (2003). L'Art contemporain est-il chrétien?, Nîmes, éd. Jacqueline Chambon.
HEARTNEY, E. (2004). Postmodern Heretics: The Catholic Imagination in Contemporary Art, New York, Midmarch Arts Press.

HESSISCHEN LANDESMUSEUMS DARMSTADT (1984). Memento Mori: der Tod als Thema der Kunst vom Mittelalter bis zur Gegenwart, cat. d'expo., Darmstadt, Hessischen Landesmuseum, 20 septembre au 28 octobre 1984, Darmstadt, Hessischen Landesmuseum.

KAEMPFER, J. (2008). "Jésus et Christ. Roman d'un couple à problèmes", dans J. KAEMPFER, Ph. KAENEL, A. BOILLAT et P. GISEL (dir.). Études de Lettres, "Points de vue sur Jésus au $\mathrm{XX}^{\mathrm{e}}$ siècle", $\mathrm{n}^{\circ} 2$, p. 111- 128 .

KAENEL, Ph. (2006). «Vera Icon: Photography and blasphemy in the XXth century», conférence au colloque Christ in Contemporary Cultures. A Cultural Studies Conference, Gordon College, Wenham (É.-U.), 28 au 30 septembre 2006, en ligne, $<$ http://www.unil.ch/webdav/site/usagesdejesus/users/ndietsch/public/vera_icon/ KAENEL_Vera_Icon.pdf $>$, consulté le 2011-09-06.

KAENEL, Ph. (2008). «Le corps du Christ entre imaginaires photographique et graphique au $\mathrm{XX}^{\mathrm{e}}$ siècle: Autour du suaire de Turin », dans J. KAEMPFER, Ph. KAENEL, A. BOILLAT et P. GISEL (dir.). Études de Lettres, "Points de vue sur Jésus au $\mathrm{XX}^{\mathrm{e}}$ siècle», $\mathrm{n}^{\circ} 2$, p. 7-27.

LIOTARD, Ph. (2003), "L'encre et le métal. Entretien avec Ron Athey», Quasimodo, $\mathrm{n}^{\circ} 7$ («Modifications corporelles»), printemps 2003, p. 113-120.

LONDON NATIONAL GALLERY (2000). Encounters; New Art from Old, cat. d'expo. de la London National Gallery, 14 juin au 17 septembre 2000, Londres, National Gallery Co.

LOTH, A. (C.1900). Le Portrait de N.-S. Jésus-Christ, d'après le Saint-Suaire de Turin, Paris, Libr. Relig. H. Oudin.

MUSÉE D’ORSAY (2002). Le Dernier Portrait, cat. d'expo. du Musée d'Orsay, Paris, 5 mars au 26 mai 2002, Paris, Éd. de la Réunion des musées nationaux.

PAULI, L. (dir.) (2006). Acting the Part: Photography as Theatre, Londres, Merrell.

PEREZ, N.N. (2002). Corpus Christi: les représentations du Christ en photographie, 1855-2002, cat. d'expo., Paris, Hôtel de Sully, Patrimoine photographique, 4 octobre 2002 au 5 janvier 2003, Jérusalem-Paris, The Israel Museum - Marval.

REAU, L. (1957). Iconographie de l'art chrétien, t. 2, Paris, PUF.

RHEIMS, B. et S. BRAMLY (1998). I.N.R.I., Paris, Albin Michel.

RUTHERFORD, P. (2000), Endless Propaganda: The Advertising of Public Goods, Toronto, University of Toronto Press.

SHAKESPEARE, W. (2002). The Tragical History of Hamlet, Prince of Denmark [c. 1604-1605], dans J.-M. DEPRATS (dir.), Tragédies, Euvres complètes I, traduction de Jean-Michel Déprats, Paris, Gallimard, La Pléiade. 
WARBURG, A. (1990). «Albert Dürer et l'Antiquité italienne", dans Essais florentins, Paris, Klincksieck, p. 159-166.

\section{Notes}

1. Voir Réau, 1957. L'auteur souligne que contrairement à la descente de croix où le cadavre est vertical, la déposition présente la dépouille à l'horizontale. Une étape chronologique a été franchie, mais aussi symbolique: Jésus est déposé sur le sol, sa dépouille gît à terre et la représentation se concentre sur le recueillement des proches, image de deuil.

2. Citons, à titre d'exemple, les deux cadavres dans des cercueils superposés peints par Marlène Dumas (1953) en 2002, dans Gelijkenis 1 en 2 (Likeness 1 and 2). Celui de la partie inférieure cite fidèlement le Christ mort de Holbein, dont on reconnaît le geste de la main, le majeur déployé et le poing serré, ainsi que les plaies et l'expression de douleur figée sur son visage. La seconde dépouille s'écarte de la version traditionnelle, même si la gestuelle est maintenue. La barbe a pourtant disparu, tout comme les stigmates, comme si un quelconque cadavre posait dans une imitatio Christi illusoire et morbide. Les jumeaux Starn (1961) reprennent quant à eux le Christ mort (avant 1654) de Philippe de Champaigne dans un photomontage de 1986, Triple Christ, nouvelle citation contemporaine d'un sujet iconographique de premier plan.

3. À ce sujet, voir notamment Grenier, 2003 ; Heartney, 2004; Perez, 2002.

4. Le processus de mise en fiction de la figure de Jésus a débuté dès la seconde moitié du $\mathrm{XIX}^{\mathrm{e}}$ siècle, lorsque ce dernier acquiert un nouveau statut qui distingue Jésus du Christ (Kaempfer, 2008). Le personnage historique s'autonomise, ce qui permet aux artistes de se libérer des canons iconographiques pour proposer une vision subjective de la figure christique. Le présent article s'inscrit dans un important projet de recherche interdisciplinaire consacré aux «usages de Jésus au $\mathrm{XX}^{\mathrm{e}}$ siècle » et mené de 2006 à 2009 à l'Université de Lausanne. Soutenu par le Fonds national suisse de la recherche scientifique (FNS), l'étude qui rassemblait les domaines de l'histoire de l'art, de la littérature et du cinéma, avait pour dessein de tisser des liens entre les différents usages de la figure christique dans le champ profane. Un colloque international a clos le projet en mai 2009. Voir Dietschy, 2011, <http ://www.unil.ch/ usagesdejesus $>$.
5. Tirage offset. Campagne de publicité Benetton de 1992. L'image ne subit que de légères modifications pour la publicité: du noir-blanc, elle passe en couleurs et le logo de la marque United Colors of Benetton est apposé sur le côté droit de l'image.

6. Statue en marbre, $174 \times 69 \mathrm{~cm}$, Rome, Vatican, Basilique Saint-Pierre.

7. Vidéo 35 mm Film/DVD, 1 minute 57 secondes, édition de 3, Londres, White Cube Gallery.

8. Voir notamment Bal, 1999; Folie et Glasmeier, 2002; London National Gallery, 2000 ; Pauli, 2006.

9. Détrempe sur toile, $68 \times 91 \mathrm{~cm}$, Milan, Pinacothèque de Brera.

10. «Le symbole de la swastika n'est ni plus, ni moins important que l'utilisation du carré, du cercle ou de l'étoile. Il est devenu depuis quelques années, un symbole lourd et haï mais je l'utilise parce que je crois réellement que c'est un symbole important et significatif qui, en lui-même, n'est coupable d'aucun crime. [...] Je voulais utiliser tous les signes afférents à tous les continents. [...] Il est surprenant qu'un tel signe, apparaissant dans de nombreuses cultures et prédominant dans de nombreux groupes ethniques, ait été utilisé pour représenter la supériorité aryenne, quand, en fait, il devrait célébrer la diversité des peuples» (Barroero, 2001).

11. C-print, $187,9 \times 152,4 \mathrm{~cm}$, édition de 6 , New York, The Proposition Gallery.

12. Les ouvrages sont nombreux sur le sujet. Voir notamment Belting, 2007 ; Kaenel, 2008.

13. Nous soulignons.

14. Détrempe vernie sur panneau de tilleul, 30,5 x $200 \mathrm{~cm}$, Bâle, Öffentliche Kunstsammlung, Kunstmuseum.

15. «Car dans ce sommeil de la mort les rêves qui peuvent surgir, / Une fois dépouillée cette enveloppe mortelle, / Arrêtent notre élan. [...] » (Shakespeare, 2002, Acte 3, scène 1, 65-67).

16. «To be, or not to be, that is the question» (Shakespeare, 2002, Acte 3, scène 1, 55).

17. À propos de la série de Bettina Rheims qui a fait scandale en représentant une femme en croix, voir Dietschy, 2008; Kaenel, 2006.

18. Rheims et Bramly, 2008.

19. Impression de pigments sur papier, $121 \times 183 \mathrm{~cm}$.

20. «[...] to die, to sleep, / To sleep, perchance to dream $[. .]$.$» (Shakespeare, 2002, Acte 3,$ scène $1,63-64)$. 